\title{
Paradoxical embolism in a boy with cystic fibrosis and a stroke
}

\author{
S D Playfor, A R Smyth
}

\begin{abstract}
An 11 year old boy with cystic fibrosis suffered a stroke, producing right sided weakness. Four years previously a totally implantable venous access device (Port-aCath) had been inserted. Magnetic resonance angiography revealed a filling defect in the left middle cerebral artery. Transoesophageal echocardiography demonstrated a thrombus attached to the tip of the Port-a-Cath and also the presence of a patent foramen ovale. After an initial period of anticoagulation the defect was closed using a septal occlusion device introduced via a cardiac catheter. The boy's neurological signs completely resolved and he remains free from further thromboembolic episodes. Whilst pulmonary embolism has been described before in relation to a totally implantable venous access device, this is believed to be the first description of a paradoxical embolism in relation to such a device.

(Thorax 1999;54:1139-1140)
\end{abstract}

Keywords: cystic fibrosis; paradoxical embolism; catheterisation

An 11 year old boy with cystic fibrosis was admitted to hospital with sudden onset of confusion and slurred speech. On examination he was found to have a right-sided hemiparesis, expressive and receptive dysphasia. He had originally presented at the age of two days with a meconium ileus and was subsequently found

Received 15 December 1997 Accepted for publication 2 March 1998

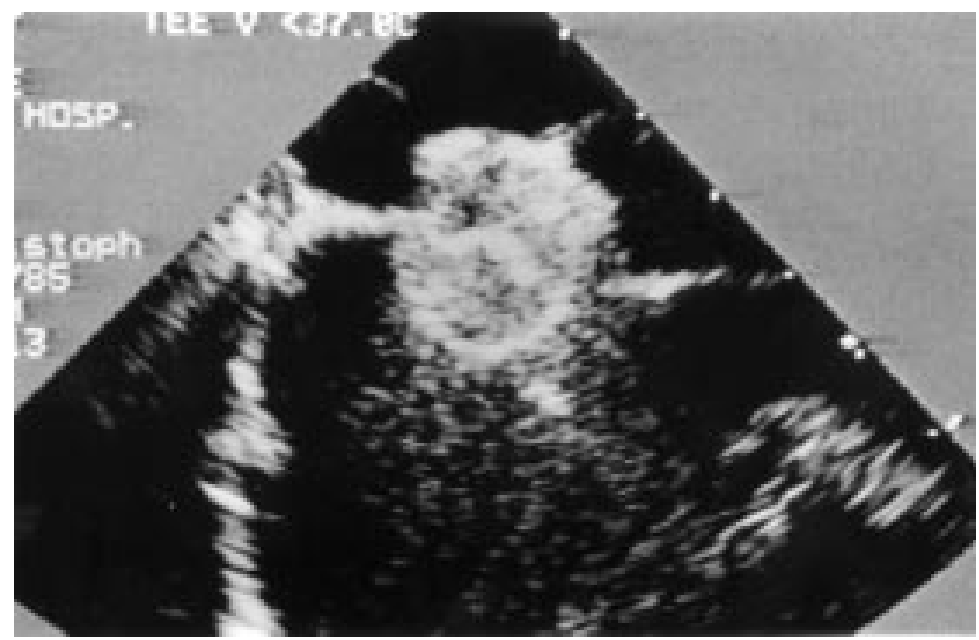

Figure 1 Septal occlusion device clamped across the atrial septum. to be homozygous for the $\Delta \mathrm{F} 508$ deletion. A sweat test performed at the age of four months confirmed the diagnosis of cystic fibrosis. Since the age of four he had required many hospital admissions for intravenous antibiotic therapy. In February 1992 a Port-a-Cath was inserted via the right internal jugular vein and secured to the anterior chest wall. His lung function was considerably compromised with a forced expiratory volume in one second $\left(\mathrm{FEV}_{1}\right)$ of $0.631(35 \%$ predicted) and a forced vital capacity (FVC) of 0.981 (49\% predicted).

A computed tomographic (CT) brain scan performed on the day after admission revealed a poorly defined area of low attenuation in the left temporoparietal region which was suggestive of ischaemia in the territory of the left middle cerebral artery. Magnetic resonance angiography confirmed recent ischaemia in this region with evidence of an embolus in the left middle cerebral artery. A transthoracic echocardiogram was performed which showed an unusual Ebstein's-like deformity of the tricuspid valve; the proximal part of the septal leaflet of the tricuspid valve was displaced apically with a point of attachment in the mid body of the right ventricle. Mild tricuspid regurgitation was also seen but no other abnormalities were apparent.

Ebstein's anomaly is associated with abnormal intra-atrial connections and therefore a transoesophageal echocardiogram was arranged. This was performed under general anaesthesia and demonstrated a small patent foramen ovale, the anterior border abutting the posterior aspect of the ascending aorta. The Port-a-Cath was seen in the right atrium and was noted to impinge against the tricuspid valve during the cardiac cycle. In addition, a large thrombus was seen attached to the tip of the Port-a-Cath in the right atrium.

Despite using intravenous contrast, no right to left shunt was demonstrable during the procedure. Techniques to enhance any right to left shunt such as coughing and the Valsalva manoeuvre were not possible because of the general anaesthetic.

The patient was anticoagulated with warfarin and started on an anti-platelet dose of aspirin. One month later the defect was closed using a $9 \mathrm{~mm}$ atrial septal occlusion device introduced via a cardiac catheter (fig 1). By this time the neurological signs had completely resolved and aspirin was discontinued after three months. There have been no further episodes of thromboembolism. 


\section{Discussion}

Paradoxical embolism was first described by Cohnheim in $1877^{1}$ but until 1930 only post-mortem cases were reported in the literature. In recent years more advanced techniques for detection of a patent foramen ovale have allowed the accurate diagnosis of presumptive paradoxical embolism during life.

Thromboembolism has been described as a complication relating to the use of totally implantable venous access devices ${ }^{2}$ but we believe this is the first time that a paradoxical embolism has been described in relation to the use of such a device.

Paradoxical embolism can only be considered proven if a venous thrombus is detected lodged in an intracardiac septal defect. This degree of certainty is extremely rare, but has been demonstrated during life using both transthoracic and transoesophageal echocardiography. ${ }^{34}$ More often we can assume paradoxical embolism if "Johnson's triad" exists-that is, (1) venous thrombosis with or without pulmonary embolism, (2) an intracardiac defect with right to left shunting, and (3) arterial embolism without a corresponding source in the left heart or proximal arterial tree. ${ }^{5}$

The reported incidence of a patent foramen ovale varies from $5 \%$ to $30 \%$. In younger adults with ischaemic stroke, where the cause may be undefined in up to $35 \%$ of cases, the incidence of a patent foramen ovale is significantly higher at around $40 \% .{ }^{6}$ This suggests that paradoxical embolism occurs more frequently than was previously thought.

Paradoxical embolism is more likely in the setting of a chronic cough, as in cystic fibrosis.
Coughing and the Valsalva manoeuvre are used during contrast echocardiography to promote right to left shunting across a patent foramen ovale, and indeed coughing is felt to be the most effective manoeuvre to demonstrate such shunting. ${ }^{7}$

Any child with an unexplained stroke requires a careful cardiological examination, particularly if there are risk factors such as an indwelling venous access device and a chronic cough. If transthoracic echocardiography shows no abnormality then more invasive investigations should be considered such as transoesophageal echocardiography.

Transcatheter techniques such as that used in our patient can offer significant advantages over open surgery in patients with cystic fibrosis. Postoperative recovery is quicker with less interruption to physiotherapy, and the avoidance of sternotomy may have important implications for any proposed heart-lung transplantation.

1 Conheim J. Thrombose und Embolie In: Vorlesungen uber Allgemeine Pathologie, Vol. 1. Berlin: Hirschwald, 1877: 134.

2 Yung B, Elborn JS, Campbell IA, et al. Thromboembolism related to a Port-a-Cath device in a patient with cystic fibrosis. Thorax 1997;52:98-9.

3 Srivastava TN, Payment MF. Paradoxical embolism: thrombus in transit through a patent foramen ovale. N Engl F Med 1997;337:681.

4 Loscalzo J. Paradoxical embolism: clinical presentation, diagnostic strategies, and theraputic options. Am Heart $\mathcal{f}$ 1986;112:141-5.

5 Johnson BJ. Paradoxical embolism. Clin Pathol 1951;4:31632.

6 Lechat P, Mas JL, Lascault G, et al. Prevalence of patent foramen ovale. N Engl f Med 1988;318:1148-52.

7 Stoddard MF, Keedy DL, Dawkins PR. The cough test is superior to the Valsalva maneuver in the delineation of right-to-left shunting through a patent foramen ovale during contrast transoesophageal echocardiography. Am Heart f 1993;125:15-9.

\section{LETTERS TO THE EDITOR}

\section{Surgery and respiratory muscles}

In their review of the effects of surgery on the respiratory muscles Siafakas and co-workers have cited my work incorrectly on two occasions. ${ }^{1}$ We reported a study of respiratory mechanics after abdominal surgery with measurements of the pressure-volume characteristics of the rib cage and abdomen. ${ }^{2}$ This report has been cited to support statements on the effects of surgery on gas exchange efficiency of the lung and of general anaesthesia on the thorax. On neither occasion is this citation appropriate. We found evidence that the abdominal muscles were active in patients after abdominal surgery, that this activity was variable, and that it could possibly explain the patterns of movement otherwise considered to represent "diaphragmatic dysfunction". These observations, along with a short review of the published work on the subject, formed an editorial ${ }^{3}$ in which I argued that the concept of diaphragmatic dysfunction was outmoded. This work has been cited in support of the effects of laparoscopic chole cystectomy, which is incorrect. Indeed, there is remarkably little direct evidence that the intercostal inspiratory muscles become more active in the patient after surgery. There is also little direct evidence in humans that the diaphragm is the respiratory muscle most affected by surgery.

Many of the changes in the shape of the thorax caused by the induction of anaesthesia can be attributed to a loss of skeletal muscle tone, with a change in the shape of the vertebral column. If this is prevented, then the changes in thoracic dimensions during induction of anaesthesia are small, variable, and do not require the hypothesis of major movements of intravascular volume as indicated in fig 1 of their review. ${ }^{4}$ This is supported by direct measurements of intravascular volume, which do not show these shifts. ${ }^{5}$

G DRUMMOND Department of Anaesthetics, The University of Edinburgh, Royal Infirmary, Lauriston Place, Edinburgh EH3 9YW, $U K$

1 Siafakas NM, Mitrouska I, Bouros D, et al. Surgery and the respiratory muscles. Thorax 1999;54:458-465

2 Nimmo AF, Drummond GB. Respiratory mechanics after abdominal surgery measured with continuous analysis of pressure, flow and continuous analysis of pressure, flow and
volume signals. Br 7 Anaesth 1996;77:317-26.

3 Dolume signals. Br f Anaesth 1996;77:317-26. Drummond GB. Diaphragmatic dysfunction: an
outmoded concept. Br f Anaesth 1998;80:27780.
4 Spens HJ, Drummond GB, Wraith PK. Changes in chest wall compartment volumes on induction of anaesthesia with eltanolone, propofol, and thiopentone. Br f Anaesth 1996;76:369-73. 5 Drummond GB, Pye DW, Annan FJ, et al. Changes in blood volume distribution between legs and trunk during halothane anaesthesia. $\mathrm{Br}$ 7 Anaesth 1995;75:707-12.

AUTHORS' REPLY We wish to thank Dr Drummond for pointing out the incorrect citation of his work in our recent review. ${ }^{1}$ We apologise for this, especially for quoting his editorial ${ }^{2}$ for the effects of laparoscopic cholecystectomy on the respiratory muscles. However, we have extensively discussed the semantics of the term "diaphragmatic dysfunction" in the methodological part of our review, sharing some of Dr Drummond's views. ${ }^{2}$ Furthermore, we have cited the work of Dr Drummond and colleagues in the general discussion of the function of respiratory muscles during surgery, arguing that respiratory muscle dysfunction, in the general sense of the term, could influence functional residual capacity. We believe that this is in agreement with Dr Drummond's findings that upper abdominal surgery alters the relative activity of respiratory muscles (including the abdominal muscles) leading to changes in the rib cage abdominal configuration (fig 1, Nimmo and Drummond ${ }^{3}$ ).

Similarly, we have cited his work to support the argument that general anaesthesia causes volume displacement between the thoracic and abdominal cavities. Dr Drummond 
argues that this could be due to the loss of skeletal muscle tone. We have no objection to this since the respiratory muscles are skeletal muscles too. Furthermore, the changes seen in the pattern of breathing after upper abdominal surgery and the relative contributions of the rib cage and abdomen during quiet breathing have been attributed by most investigators to dysfunction of the diaphragm. ${ }^{4-6}$ Dr Drummond's experiments offer another explanation, pointing out the role of the abdominal muscles (the major expiratory muscles). Our own experiments have shown that upper abdominal surgery impairs the global strength of the inspiratory and expiratory muscles to produce maximum pressure. ${ }^{78}$ It is well known that the major contributor to the maximum inspiratory pressure (MIP) is the pressure produced by the diaphragm. Thus, it is logical to suggest that, if MIP is grossly reduced after surgery, this is primarily due to diaphragmatic dysfunction. We believe that the above differences may be due to the different experimental conditions (quiet breathing versus maximum static pressures).

N M SIAFAKAS

I MITROUSKA D BOUROS

D GEORGOPOULOS

Pulmonary Department,

University of Crete Medical School, 71110 Heraklion, Crete, Greece

1 Siafakas NM, Mitrouska I, Bouros D, et al. Surgery and the respiratory muscles. Thorax 1999;54:458-465

2 Drummond GB. Diaphragmatic dysfunction an outmoded concept. Br f Anaesth 1998;80: 277-9.

3 Nimmo AF, Drummond GB. Respiratory mechanics after abdominal surgery with continuous analysis of pressure, flow and volume signals. Br F Anaesth 1996;77:317-26.

4 Ford GT, Whitelaw WA, Rosinal WT, et al. Diaphragm function after abdominal surgery in humans. Am Rev Respir Dis 1983;127:431-6.

5 Simonneau G, Vivien V, Sartene R, et al. Diaphragmatic dysfunction induced by upper abdominal surgery: role of postoperative pain. Am Rev Respir Dis 1983;128:899-903.

6 Ford GT, Rosenal TW, Clergue F, et al. Respiratory physiology in upper abdominal surgery. tory physiology in upper abdom
Clin Chest Med 1993;14:237-52.

7 Siafakas NM, Stoubou A, Stathopoulou M, et al. Effect of aminophylline on respiratory muscle strength after upper abdominal surgery: a cle strength after upper abdominal surgery:
double blind study. Thorax 1993;48:693-7.

double blind study. Thorax 1993;48:693-7.
Rovina N, Bouros D, Tzanakis N, et al. Effects of laparoscopic cholecystectomy on global respiratory muscle strength. Am $\mathcal{F}$ Respir Crit Care Med 1996;153:458-61.

\section{Postoperative air leaks}

The study by Waller et $a l^{1}$ did not attempt to compare the clinical benefit, cost effectiveness, or relative convenience of the two alternative chest drainage systems - a flutter valve drainage bag or the conventional underwater seal Instead it claims that the flutter valve offers "a physiologically more effective" alternative to the underwater system. However, a pneumothorax is a pathological rather than a physiological condition in which it is imperative to remove air from the pleural cavity. In this situation the pleural pressures will be higher than in the normal physiological state and, indeed, air will only exit the pleural cavity via a chest drain when the pleural pressure exceeds atmospheric pressure. Any drainage system for a pneumothorax that renders the pleural pressure more negative (relative to the atmospheric pressure) will therefore reduce the exit of air, other than by more forcible expiration, and reduce its effectiveness.
A further theoretical but serious consideration is that of re-expansion pulmonary oedema (REPE). This has been associated with excessively negative pleural pressures after removal of air $^{2}$ or fluid ${ }^{3}$ from the pleural space.

The study concludes that an ideal postoperative drainage system will "allow the maintenance of a negative intrapleural pressure, even in the presence of an air leak". In our view a negative pleural pressure in this situation would only serve to encourage the egress of more air from the damaged lung surface into the pleural cavity, thus worsening the situation.

Whilst accepting that the postoperative situation may differ somewhat from that which pertains in a spontaneous pneumothorax because of the presence of fluid/blood in the pleural cavity, we would expect the same physiological principles to apply, especially in the presence of an air leak. There is a risk that the conclusions from this paper might be extrapolated into general respiratory practice and the scenario of spontaneous pneumothorax. For all of the above reasons we would urge caution before abandoning the standard and well tried technique of drainage of the pleural cavity (underwater seal) in favour of the suggested alternative, at least before data from larger, controlled clinical trials become available.

M HENRY Medical Chest Unit, Castle Hill Hospital, Cottingham,

North Humberside HU16 57Q,

J E HARVEY Southmead Hospital, Bristol BS10 5NB,

$U K$

A G ARNOLD Medical Chest Unit, Castle Hill Hospital, Cottingham,

North Humberside HU16 $57 Q$,

1 Waller DA, Edwards JG, Rajesh PB. A physiological comparison of flutter valve drainage bags and underwater seal systems for postoperative leaks. Thorax 1999;54:442-3.

2 Matsuura Y, Momimura T, Murakami H, et al. Clinical analysis of re-expansion pulmonary oedema. Chest 1991;100:1562-6.

3 Light RW. Pneumothorax. In: Retford DC, ed. Pleural diseases. 3rd edn. Baltimore, USA: Williams and Wilkins, 1995: 267-9.

AUTHORS' REPLY We thank Dr Henry and colleagues for their comments regarding our study. We must first emphasise that we were not intending to make any conclusions regarding the relative clinical or financial benefits of either drainage system, and that we were specifically addressing the situation of postoperative air leaks. We have shown that, at resting tidal volume, flutter valve drainage bags maintain a more negative (but not excessively negative) intrapleural pressure than underwater seal systems and therefore are more likely to restore the normal physiology of the pleural space.

The aim of chest drainage in this situation is to restore and maintain the negative intrathoracic pressure necessary for lung re-expansion which will allow reactivation of the surface forces promoting visceral and parietal pleural apposition. ${ }^{1}$ This is dependent on removing excess gas or fluid from the pleural space. We cannot therefore agree with the assertion of Dr Henry and colleagues that achieving negative intrapleural pressure will reduce the exit of air (by which we assume they mean will reduce lung expansion). While it has been shown that excessive pleural suction in the presence of a high flow bronchopleural fistula may perpetuate air leakage, ${ }^{2}$ Henry et al are ill advised to extrapolate this principle to the more common clinical scenario of a smaller volume air leak.

Re-expansion pulmonary oedema is a recognised but uncommon complication of chest drainage which reflects the rapidity of lung re-expansion. ${ }^{3}$ This is rare in prolonged chest drainage for persistent air leakage and the concerns of Dr Henry and colleagues cannot be justified.

We welcome the opportunity to discuss this common but seldom debated area of respiratory practice. The standard and well tried method of underwater seal drainage is a source of many misunderstandings and complications. ${ }^{4}$ The Heimlich flutter valve, whose physics we have studied, has been shown to be clinically effective by Graham $e t$ $a \bar{P}$ and others and further data from a randomised clinical trial in prolonged air leakage will soon be forthcoming.

DAVID A WALLER JOHN G EDWARDS

Department of Thoracic Surgery, Glenfield Hospital, Leicester LE3 9QP, $U K$

PALA B RAJESH Department of Thoracic Surgery, Heartlands Hospital, Birmingham, UK

1 Hyde J, Sykes T, Graham TR. Reducing morbidity from chest drains. BMF 1997;314:914-5.

2 Powner DJ, Cline CD, Rodman CH. Effect of chest tube suction in gas flow through a bronchopleural fistula. Crit Care Med 1985;13:99101

3 Waller DA, Saunders NR. Unilateral pulmonary oedema following removal of a giant pleural tumour. Thorax 1989;44:682-3.

4 Harriss DR, Graham TR. Management of intercostal drains. Br f Hosp Med 1991;45:383-6.

5 Graham ANJ, Cosgrove AP, Gibbons JRP, et al. Randomised clinical trial of chest drainage systems. Thorax 1992;47:461-2.

\section{Rare diseases}

We welcome the series of articles on rare pulmonary disorders which started in the March 1999 issue of Thorax. In the introduction to the series Tattersfield and Du Bois ${ }^{1}$ describe the frustrations felt by patients unable to obtain information about their rare pulmonary condition from clinicians with little or no experience of that disorder. The isolation of such patients is emphasised when they are unable to share their experiences with others diagnosed with the same disorder. Whilst we appreciate that this is true for many patients, we would draw attention to the massive range of information on rare pulmonary disorders available to both patients and doctors via the Internet. There are over three million UK subscribers to the Internet currently and, with the advent of digital television, this number will rise exponentially over the next few years. The problem then facing the patient (and clinician) is too much information. For example, lymphangioleiomyomatosis, the subject of the first article in the series, has over 500 Internet sites accessible worldwide to patients. We hope to provide assistance to patients who may lack confidence in knowing where to turn for information relevant to their needs. 
The North East Thames Training Programme for Specialist Registrars has run an Internet based educational programme for medical trainees for over two years. Using this experience, trainees and trainers are now opening a website for patients with rare pulmonary disorders. The group is evaluating existing websites for validity of medical information and the relevance of presentation and content for patient rather than medical needs. It is hoped that the resulting reference site will index all available Internet information and allow patients to access information that is both reliable and relevant. To achieve this goal we are working with the help of patient support groups based with the British Lung Foundation, Organising Medical Networked Information (OMNI), and the Oxford University and British Library health care information team.

This is an enormous project which we feel will have increasing importance with time. We have already received offers of help from registrars in Wales, Scotland, and Australia. The North East Thames registrars welcome offers of co-operation from other similar groups.

C M ROBERTS Programme Director North Thames East Respiratory Medicine STC Whipps Cross Hospital, London E11 1NR on behalf of the North Thames (East) respiratory trainees

1 Tattersfield AE, du Bois RM. Rare diseases: introduction. Thorax 1999;54:253.

AUTHORS' REPLY We agree with Dr Roberts that the Internet raises special issues for patients with rare diseases and that the problem is often too much information rather than too little. With respect to lymphangioleiomyomatosis (LAM), our approach in Nottingham has been to produce a four page fact sheet for patients which we have given to patients in the LAM Trust and to some of the doctors looking after the patients. The fact sheet was piloted amongst a few patients with LAM and modified in the light of the feedback we received. Several patients have obtained the fact sheet from the LAM Foundation in the USA which goes into slightly more detail than our document, particularly with respect to prognosis; as might be expected, some patients appreciate the further information whilst others do not.

Whether specialist registrar trainees should be deciding what patients with a rare disease should be encouraged to read is more debatable since some will never have met a patient with the disease in question. It seems more appropriate to us for fact sheets and information to be provided by people with some knowledge and expertise of managing patients with the particular disease, in conjunction with the patients themselves. The British Thoracic Society is planning to develop an orphan disease register and to encourage research into rare disorders. One of their remits will be to consider what information is required and how it should be presented and piloted.

A E TATTERSFIELD City Hospital, Nottingham NG5 1PB, $U K$

R M DU BOIS

Royal Brompton Hospital, London SW3 6NI

\section{Aspergillus fumigatus: re-invention of the wheel}

The paper by Murayama et $a l^{1}$ contains the unwary statement that their study may have been the first to demonstrate the suppressive effects of Aspergillus products on antifungal host defences by both human alveolar macrophages and PMNs. A search for Aspergillus in the eThorax website would have saved both them and your referees from accepting this. In a series of papers published in the $1980 \mathrm{~s}$ Maura Robertson and I showed this in animal and human cells, demonstrated the paradoxical effect of complement and, perhaps importantly, showed that the substance produced by the spores had similar effects on soil protozoa, thus explaining the biological paradox as to why an organism that gains nothing from colonising animal lungs should have developed such exquisite antiphagocytic properties. Some of this work was summarised in our paper pupblished in the Lancet in 1989. ${ }^{2}$ These effects are discussed in at least one well known textbook of respiratory disease.

It is to be hoped that the "wheel reinventing" tendency of authors who search the literature back only five years and the short memory or ignorance of referees may be alleviated by use of your new web site.

ANTHONY SEATON

Department of Environmental and Occupational Medicine,

University of Aberdeen Medical School, Foresterhill,

Aberdeen AB25 2ZD,

$U K$

1 Murayama T, Amitani R, Ikegami Y, et al. Effects of Aspergillus fumigatus culture filtrate on antifungal activity of human phagocytes in vitro. Thorax 1998;53:975-8.

2 Seaton A, Robertson MD. Aspergillus, asthma and amoebae. Lancet 1989;i:893-4.

3 Seaton A, Seaton D, Leitch AG. Crofton and Douglas's Respiratory Diseases (4th ed). Oxford: Blackwell Scientific, 1989.

AUTHORS' REPLY We thank the editors for the opportunity to respond to the concerns raised by Professor Seaton. His group ${ }^{1-4}$ and Drs Mullbacher, Eichner et al in Australia ${ }^{5}{ }^{6}$ are pioneers in studies of the antiphagocytic activity of Aspergillus derived products and they have done some excellent work. We cited some of their original articles in our previous paper. Professor Seaton et al have shown that spores and spore diffusates of $A$ fumigatus suppress the production of superoxide anion and hydrogen peroxide by rodent phagocytic cells ${ }^{1}$ and that diffusates of $A$ fumigatus spores inhibit phagocytosis of antibody coated radiolabelled sheep red blood cells by mouse peritoneal exudate cells. $^{2}$ They have also performed experiments using human cells and have shown that spore diffusates of $A$ fumigatus reduce the migration of human polymorphonuclear leucocytes and decrease the capacity of mouse peritoneal exudate cells to spread on glass. ${ }^{3}$ They have measured the ability of human pulmonary macrophages to bind and kill $A$ fumigatus spores and have examined the effects on killing of heat labile serum components. ${ }^{4}$ However, they have not presented data on the suppressive effects of Aspergillus derived products on polymorphonuclear leucocyte mediated damage to $A$ fumigatus.

It has been considered that selective protection against Aspergillus spores by mononuclear phagocytes and against hyphae of Aspergillus by polymorphonuclear phagocytes are critical host defences in humans. ${ }^{8}$ Since Aspergillus spores enter mainly through the respiratory tract and proliferate in mycelial form in human tissues, our study has focused on interactions between Aspergillus spores and human alveolar macrophages, and also between Aspergillus hyphae and human polymorphonuclear leucocytes. In our study ${ }^{9}$ we showed that $A$ fumigatus culture filtrate suppressed not only human polymorphonuclear leucocyte mediated $A$ fumigatus hyphal damage but also inhibition of spore germination of $A$ fumigatus by human alveolar macrophages, and suggested that $A$ fumigatus produces a variety of substances, some of which may suppress antifungal (anti- $A$ fumigatus) activity of human alveolar macrophages and polymorphonuclear leucocytes. To our knowledge, our study is the first to demonstrate the suppressive effects of Aspergillus derived products on human polymorphonuclear leucocyte mediated hyphal damage of Aspergillus and also on inhibition of spore germination of Aspergillus by human alveolar macrophages.

TAKOKO MURAYAMA
RYOICHI AMITANI
Department of Infection and Inflammation,
Chest Disease Research Institute,
Kyoto University,
Kyoto,
fapan

1 Robertson MD, Seaton A, Milne LJR, et al. Suppression of host defences by Aspergillus fumigatus. Thorax 1987;42:19-25.

2 Robertson MD, Seaton A, Milne LJR, et al. Resistance of spores of Aspergillus fumigatus to ingestion by phagocytic cells. Thorax 1987;42: 466-72.

3 Robertson MD, Seaton A, Raeburn JA, et al. Inhibition of phagocyte migration and spreading by spore diffusates of Aspergillus fumigatus. $\mathcal{F}$ Med Vet Mycol 1989;27:295-302.

4 Robertson MD, Kerr KM, Seaton A, et al. Killing of Aspergillus fumigatus spores by human lung macrophages: a paradoxical effect of heat labile serum components. $f \mathrm{Med}$ Vet Mycol 1989;27:295-302.

5 Mullbacher A, Waring P, Eichner RD, et al. Identification of an agent in cultures of
Aspergillus displaying antiphagocytic and Aspergillus displaying antiphagocytic and Microbiol 1985;131:1251-8.

6 Eichner RD, Salami MAL, Wood PR. The effect of gliotoxin upon macrophage function. Int $\mathcal{F}$ Immunopharmacol 1986;8:789-97.

7 Murayama T, Amitani R, Ikegami Y, et al. Suppressive effects of Aspergillus fumigatus culture filtrates on human alveolar macrophages and polymorphonuclear leukocytes. Eur Respir 7 1996;9:293-300.

8 Schaffner A, Douglas H, Brude A. Selective protection against conidia by mononuclear and against mycelia by polymorphonuclear phagocytes in resistance to Aspergillus. F Clin Invest 1982;69:617-31.

9 Murayama A, Amitani R, Ikegami Y, et al. Effects of Aspergillus fumigatus culture filtrate on antifungal activity of human phagocytes in vitro. Thorax 1998;53:975-8.

\section{NOTICE}

\section{The Dr H M (Bill) Foreman Memorial Fund}

The Trustees of the Dr H M (Bill) Foreman Memorial Fund invite applications for grants relating to study in respiratory disease. Limited funds are available for registered medical practitioners to assist in travelling to countries other than their own to study respiratory disease, and also for support for clinical research abroad. Intending applicants should write for further details to Dr Brian $\mathrm{H}$ Davies, Llandough Hospital, Penarth, Vale of Glamorgan CF64 2XX, UK. 